\title{
Multi-Platform Inter-APP Communication Solution for IOT Services Implementation
}

\author{
Dr. Gasim Alandjani \\ Information and Computer Technology Department, Yanbu University College,Kingdom of Saudi Arabia
}

\begin{abstract}
Technology has totally changed the culture and working of people. With every passing day it is gaining popularity and becoming one of the most important gadgets of our lives. Researchers are enthusiastically pursuing areas of research which can contribute to Internet of Things (IoT). Now a day's sensing communication, control and actuation is becoming more refined and available everywhere, there is momentous overlap in these communities, still there are some missing gaps that need to be filled and addressed for better understanding of communication among these real life objects. The paper give an overview for inter app communication for multi-platforms users who will be using different IOT Services .
\end{abstract}

Keywords: IOT, Smart City, Smart Homes, Virtual Architecture, IOT Stack, Smart Devices, Sensors, Smart Vehicle.

\section{Introduction}

Current era of Information technology is introducing latest technology gadgets with every passing day IoT (internet of Things) is one of them. It is considered as the network of physical objects including electronic devices, vehicles, smart building, smart home appliances and many other real life objects which have been embedded with network connectivity, with the help of software and sensors to exchange or share data for some intelligent processing. It provides the opportunity to share data anywhere with anytime manner needs open, scalable, secure and standardized infrastructure which don't exit fully today. This is mainly focusing on Technology rather mainly focusing on other relatedareas [1].

We can't correlate ubiquitous computing, Internet Protocol, communication technology, embedded devices, its applications rather it's a bigger picture by enabling even social websites. Internet of people, internet of devices. Infect it provides a combined flavor of all these technologies. Some IoT projects are EPoSS which provides sustainable structure for smart system integration. BRIDGE project was developed for Radio Frequency Identification (RFID) and EPC global Network which identifies items via Electronic Product Code (EPC). Do-it-Yourself Smart Experience project (DiYSE) was intended to motivate common people to develop and install and manage applications to convert their normal living in smart environment. Further projects including Internet of things architecture (IoT-A) provide the opportunity for business-based internet of things, Experimental Living Labs for Internet of Things (ELLIOT) and Internet of things at work have started their work with the promise that they will further extend their work and will contribute to the research on IoT in Europe[3].

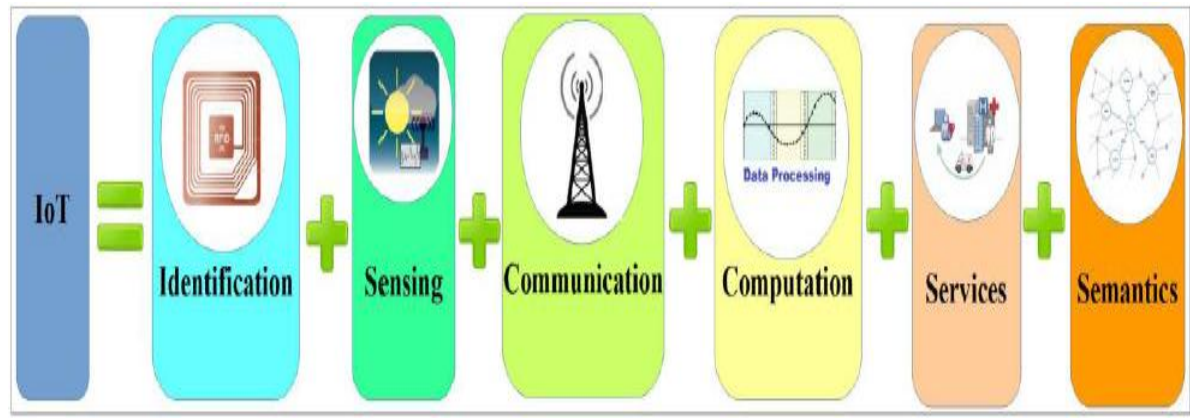

Figure 1: IOT Building Blocks

Here are few guidelines which can be used as catalyst for successful contribution to internet of things sometimes we use to call them 4Es of IoT.

1) Envision: IoT should provide holistic scenarios focusing on private, business and social benefits.

2) Extend: It should leverage existing developments and technologies.

3) Enable: It should have ability to solve adaptation challenges like privacy, security and confidentiality, invent new mechanism for sharing cost and benefits. 
4) Evaluate: Proper discussion should be done among stakeholders for New approaches to make them user centric.

\section{IOT Application and Challenges}

If we compare applications with challenges with social needs and developments to enable major technologies includingnano-electronics and cyber physical system to which face challenges from many different aspects.

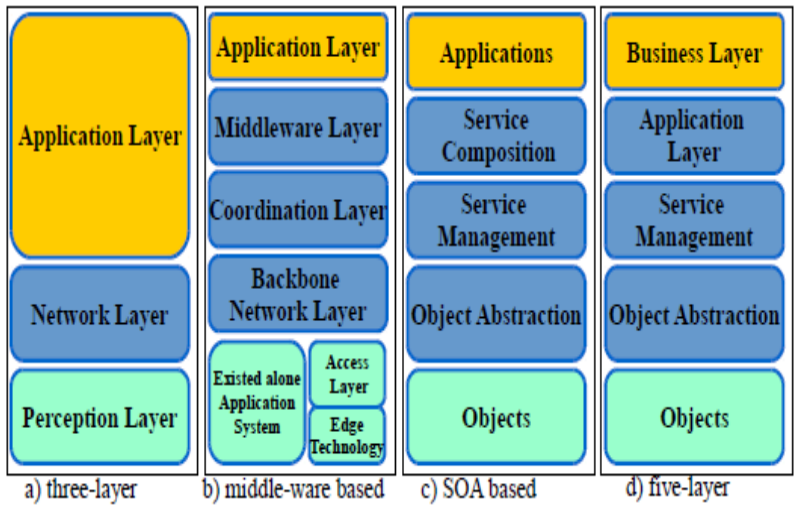

Figure 2: IOT Layered Architecture

In Smart Cities high quality of life is available supported by some sustainable services including environment, mobility and economy. Although several challenges are there but still ITC Infrastructure is the backbone for the extensive development in these areas:

- People should be trained to use some cutting-edge technologies for smart and better services instead of relying on traditional procedures.

- Information gathered by sensors can be described by following schemes or by creating algorithms for exchange of information in a productive and useful manner among different City services for residents.

- To design a mechanism for cost effective deployment and Installations of such sensors.

- Ensuring reliable readings for huge amount of sensors used by different objects.

- Preference should be given to the algorithm and protocols which use less energy but also main focus should also be on a bigger picture for processing and analysis of data for the whole city. Which will ultimately be helpful for the large scale deployment of IOT and its integration.

\section{Smart Grid and Smart Energy}

With every passing day there is increase in urbanization which eventually increased demand for energy and there is more awareness about the supply, consumption and infrastructure. Energy resources will no longer be based upon fossils or nuclear resources. As aresult, renewable energy and alternative sources of energy are gaining popularity which ultimately need a flexible and intelligent grid where power fluctuations can be controlled in the source and sinks by suitable reconfigurations. All such functions can be achieved by network intelligence devices such as devices, appliances, micro generation equipment, and grid infrastructure and consumer products. Future energy grids should have medium and small power plants and sources of energy can be combined virtually and incase of disaster in any particular area they can be made isolated from the grid system so as not to disturb the services in adjustment areas $[16,17]$.

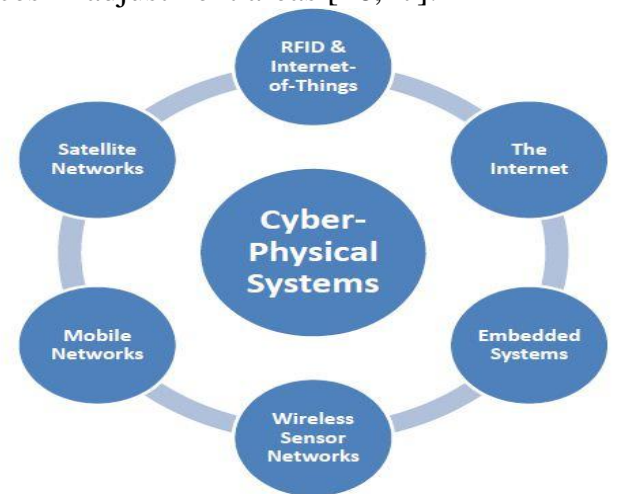

Figure 3: Large Scale Cyber-Physical Systems Components 
If we consider the major challenges we can rank cyber-physical system's integration with existing grid system as a biggest challenge.Through smart grid efficiently transmission of energy is possible by using smart transmission concept from distributed to concentrated power plants by considering standard security standards.

So the smart grid is like implementation of 'Internet' in which energy packetsaretreated just like a data packet. So concept internet of energy (IoE) is used. It provides power distribution, storage of energy and management of grid concept in an innovative way. It will provide the vast range of energy storage and monitoring from local devices to international level devices. Smart metering is one of the real life example for this kind of energy management which provide information for users and help them to better plan and manage their energy requirements as per their needs with much more efficiency to reduce the wastage level of energy for end users and to facilitate more consumers. In order to facilitate the communication between different vendor products protocol stack is needed. Depending upon connectivity Cloud based IoT concepts may be advantageous. Transfer of information and energy will be controlled and monitor by energy gateways. Here are some challenges to achieve smart energy grid:

- Communication among network elements should be in a very safe and secure environment by considering all the standard security measures.

- Interoperability and scalability should also be addressed.

- Efficient, reliable and robust smart sensors should be used for energy saving.

- Technologies for data obscuritywith major concern on privacy of individual and public data should be ensured.

- Critical and delicate latencies should be dealt with professional honesty.

- System partitioning based upon local or cloud based information with intelligence.

- Proper use of filters and mining should be ensured while processing data in bulk quantity at large scale level.

- Reliable internetworking of heterogeneous systems should be doing by real-time design methods and models.

- Self-healing and damage control system concept should be implemented and its factions should also be scalable.

- In case of fluctuation during the process of electric supply of energy from alternative energy sources (including wind forms and solar grid stations)power grids should respond accurately and in timely fashion.

\section{Smart Transportation}

Transportation has been considered as backbone for life on earth since ages and it's even true in our modern life of technology. Internet of Energy and Internet of Vehicles are the same concepts and working on the same parameters to eventually support the future trends of smart mobility and transportation. To design and develop a human behavior centric and antonymous vehicle is still a challenge and still its difficult to predict the behavior of human drivers who are driving vehicles on the roads in a mixed traffic environment. On the other side if we talk about the implementation of IOT by considering telematics and automotive for implementation of this kind of system we can easily drive the following suggestions. [18]

- We should also be consider the fact in mind to define standards for voltage charging of power electronic systems and recharging them and they must be controlled by system within the vehicle.

- Components of bidirectional operation and also for electricity needs flexible billing should be considered.

IoT has already been used for controlling and monitoring the functions of vehicles in real world environment like Certain technical functions of vehicles are monitored on the garage or the maintenance center. Data from sensors is collected by small on board unit and communicated to the service center via internet.Vehicles organize themselves for better energy used and better follow of traffic avoiding deadlocks. Mutual interaction among vehicles and with the infrastructure provides safety while reduces number of accidents [19].

IoT provides multi-modal transport system sense the traffic intelligently and accordingly make optimized route plans to avoid any congestion of traffic in some particular area and even by sharing information with adjacent vehicles to opt the alternative routes in case if the some congestion or accident on some particular route. This will optimize the smooth flow of traffic with least hurdles and which eventually will be biggest advantage of IoT by smart use of cutting-edge technologies. 


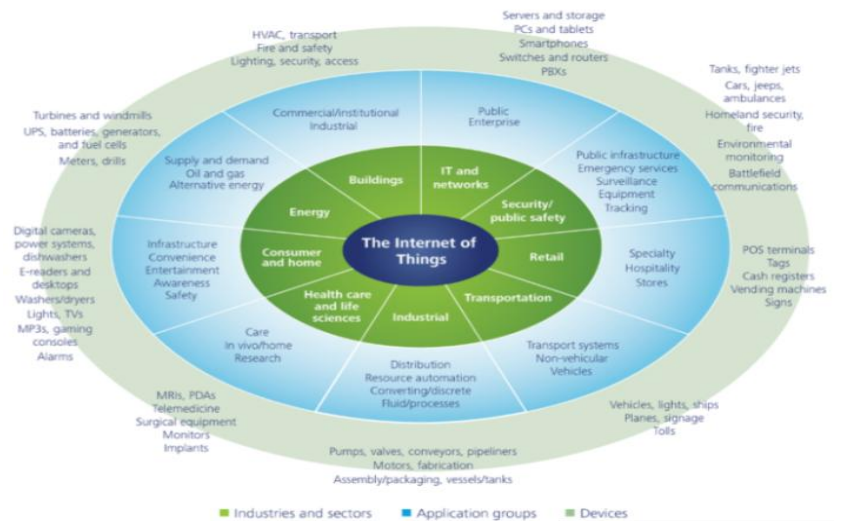

Figure 4: Basic Building Blocks for IOT[5]

During process of collecting information related to roads, traffic and weather condition very robust sensors are required [5]. Which require sophistication in data mining techniques apart from all the some challenges are also to be addressed with top priority.

- At network edges and inter vehicle there is a great need for secure communication

- Smart, Robust and reliable sensors should be used in vehicles.

- Individual and business Privacy should be considered at a top priority for the privacy of data.

- During the transfer of data with in infrastructure its privacy should be considered at a top priority.

\section{1) Smart Infrastructure\& Homes:}

Where Technology Development has made many advancements and modifications in living style of people it also made several new trends in design of building and infrastructure for modern era commercial buildings and homes. Where smart homes and smart building concept is very popular[21,22]. Many commercial firms are offering different design scheme and building maps to equip them with technology that support a design in such a way that all the electronics devices and appliances should be controlled with a single controller and by the click of single button with addition to add health devices to support special services for better health and environment facilities under the same single building with better monitoring gadgets to monitor and control energy and intelligent systems which monitor better environment by the use of these sensor monitor system with the help of smart metering and smart heating, ventilation and air conditioning (HVAC) with the help of smart sensors to keep temperature of building to a specific level or modify it as per day to day or event to event requirement of building .[5,24]

Smart devices including touch screen, 3D browsers or even smart phones screens could be used to check status of all these kind of information. Sensor based data is processed by cloud hosted software. Smart building concept can be divided into three layers

(i) Envelope: It deals with building structure including Green materials, design and thermal structure.

(ii) Connectivity: it deals with building operation controls through sensors, actuators and telecom infrastructure.

(iii) Software: it involves building operations optimization. Its main components are visualization, analytics and data storage.

There are different integrated services that are provided by these layers subsystems like lightening, power and electricity, HVAC, security, water and waste management. Integration of cyber physical system with both buildings and outer entity like electrical grid requires stakeholders' cooperation to achieve interoperability. Architecture of building automation system is divided into three levels [15].

(iv) Field Level: Devices access and data distribution.

(v) Automation Level: Rules Distribution and Rules Engine

(vi) Management Level: Algorithm distribution and composition.

\section{2) Smart Factories \&Manufacturing:}

IoT allows access to devices and machines in the manufacturing system. It has digitized the manufacturing system. It allows factory to its running applications around like sharing of production facilities as a service by establishing connection with smart grid. Smart factory can access external stakeholders like suppliers of production, logistics, maintenance and retooling actors. Enterprisesare making huge use of available data, RFID, M2M, business analytics, sensor technology GPS, cloud services, embedded technology, mobility, ID detection technology, physical and virtual security, wireless network and standardization. With all these intended features that we will be getting at one side there some of the challenges which are associated and can 
be noted as network integration, and interoperability and system affordabilityof such a multifaceted and multi featured systems [7].

\section{3) Smart Health:}

Health monitoring devices are application specific, non-interoperable and diverse in architecture. The IoT can be used in clinics to monitor the critical patients. Sensors will collect the comprehensive physiological information, use clouds and gateways to analyze data and send the data wirelessly to the caregivers. IoT is also playing very prominent role in the field of medicine by preventing and managing chronic diseases. There are many challenges in cyber-physical network like hardware, connectivity, software development and communication.

Latest novel technologies are required todeal with trends on wired/wireless communication having medium to high speed interfaces, optimality and modular design for multifaceted and smart integrated devices [10].

IoT applications Process can be summarized as

- There must be standardization of sensors and MEME interface for an open platform to maintain the quality and create competition among manufacturers of bio- medical devices.

- Information gathering and their processing should be highly automated.

- Real-time data availability should be there for caregivers anywhere with appropriate software and privileges

- Intelligent and smart devices can be re-used for data travelling over trusted web.

- Data should beinterchangeable within clinical pathways, home, ambulance, clinic and hospital with some very reliable and automated system.

\section{4) Smart Logistics And Retail:}

Retail Sector is also one of the major sector for getting the main benefits of IoT where it provides proficient solutions by addressing the right content for a right person at right time and selecting the right place. Acclimatizing to the priorities and changing tastes of people will be vital task for retailers all over the world. To meet and remain updated with all these changes they must use smart and efficient connected devices. They are using sensors and scannersin addition to other IoT technologies to optimize all their operations and getting surety of delivery of their respective products from production unit till door step of consumer. They can also make a better use of Meta data to help them for getting a clear picture through data of their products, employees and other stakeholders and possible option to bring them all on a single platform for getting much better results by putting less efforts and time with optimal outcomes [11].

\section{5) Security For Food And By Tracking:}

Being one of the basic element for life, food and water are at top of the list for life on planet which shows their importance for existence of all especially for human being. Production of Organic foods without further addition of chemical substances will be valued. Similarly fresh water is also important for daily life. IoT can be used for the tracking of any consignment from production unit to consumerplace[14]. IoT applications need development of frameworkwith assurance of the following points.

- The things connected to IoT should provide valued services to the larger system

- System should provide APIs that that allow users take advantage of system suitable to their needs and developers to innovate something new from provided data and services

- Security should be maintained to prevent system from attacks

There are also some challenges to achieve smart goals like

- Design of secure, cost-effective and tamper-proof mechanism for tracking goods (water and food) from productionunit to consumers.

- providing sufficient information and confidence to consumers in production process monitoring and providing details of production process with security

- Ensuring secure data exchange with confidentiality and trust among infrastructure and different applications to prevent false information which will affect health of consumers and can create financial consequences stakeholders.

\section{6) Participatory Sensing:}

People living in a any community wanted to be facilitated in several different ways by make ease in different activities like finding their favorite food offered by some of their favorite restaurant, competent and honest car mechanic, favorite movie theater etc. all these kind of services are offered by a layered technology 
approach by IoT. Following figure 5 presents a comparison between traditional network stake and IoT Network stack.

\begin{tabular}{|l|l|l|}
\hline & & Application \\
\hline TCP/UDP & UDP & Transport \\
\hline \multirow{2}{*}{ EP } & IPV6 & Network \\
\cline { 2 - 3 } & 6LowPAN & $\begin{array}{l}\text { Data Link \& } \\
\text { Physical }\end{array}$ \\
\hline $\begin{array}{l}\text { Traditional } \\
\text { Network Stack }\end{array}$ & $\begin{array}{l}\text { IETE 802.15.4 Network } \\
\text { Stack }\end{array}$ \\
\hline
\end{tabular}

Figure 5: IOT VS Traditional Network Stack

The development in IoT has made it easier to facilitate people lives. Smart phones are made up of lots of actuators and sensors like microphone, speakers, camera and temperature gauge etc. Participatory sensing application utilizes each person's mobile phone and car with automatic sensory stations that take multiple snapshots of the immediate environment [15].

- Design of the algorithm with given same observations at different conditions

- Design a robust mechanism for processing and of observations and data analysis in real time.

- Reliability \& trustworthiness of observed data by proper identification and authentication of data sources

- Ensuring privacy of individuals while providing observations

- Addressing scalability and large scale deployments.

7) Missing Dots:

Social, economic and technical implications have to be thoroughly considered in order to have complete deployment of IoT. Atzori came up with comprehensive IoT paradigm that classified the concept of technologies and standards through the convergence of Internet, Things and Semantic visions (Figure 6) [1]. Later, Tereffy et al. proposed to modify Atzori to serve human-centered prospective of IoT [2]. Figure 7 illustratesTereffy's framework that serves as a design tool for HCI audience with the following findings:

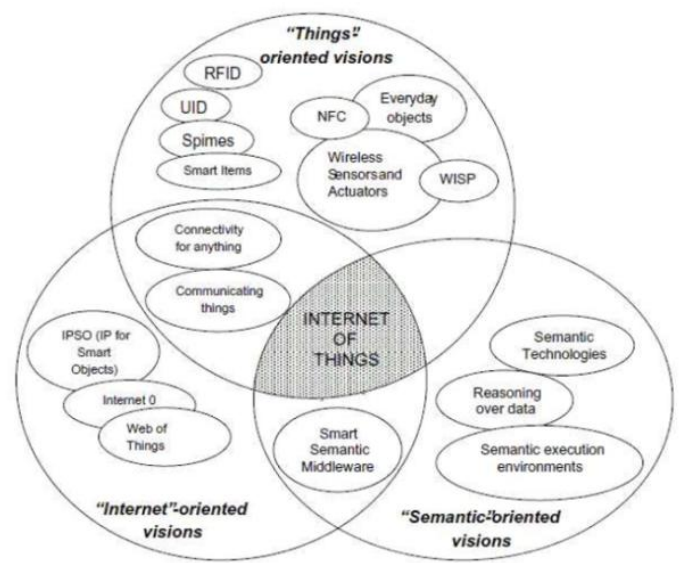

Figure 6: Connecting Dots [1]

(i) HCI hasn't covered well in Things / Semantic category in Atzori paradigm

(ii) Paper identified the Internet category as out of scope of HCI. This can be investigated further or studied it in the "Internet" point of view

(iii) The only design approach can be used with HCI is the Participatory design. Building a design using this HCI concept can be a great opportunity for future research work. 


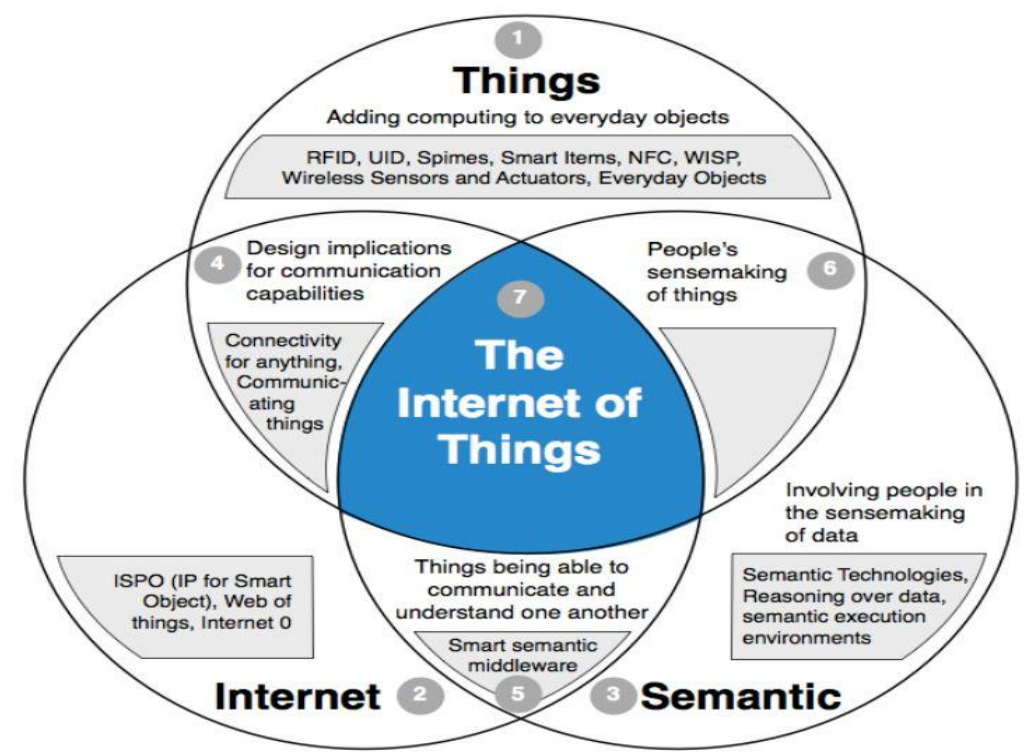

Figure 7: Dot Connection Point [2]

Following Table (Table 1) shows the comparison Atzori paradigm and Tereffy's framework and illustrate the transformation.

\begin{tabular}{|c|c|c|}
\hline Category & Atzori's framework & $\begin{array}{l}\text { Modified framework } \\
\text { (Tereffy's } \\
\text { framework) }\end{array}$ \\
\hline Things & $\begin{array}{l}\text { Physical components (RFID, } \\
\text { NFC,etc) }\end{array}$ & $\begin{array}{l}\text { Adding computing to } \\
\text { everyday objects }\end{array}$ \\
\hline Internet & $\begin{array}{l}\text { Protocols and language to } \\
\text { communicate between objects }\end{array}$ & Beyond HCI Scope \\
\hline Semantic & $\begin{array}{l}\text { Technologies: process, store } \\
\& \text { use data generated by } \\
\text { Internet of things }\end{array}$ & $\begin{array}{l}\text { Involve people in the } \\
\text { sense making of data }\end{array}$ \\
\hline $\begin{array}{l}\text { Things / } \\
\text { Internet }\end{array}$ & $\begin{array}{l}\text { Connectivity } \\
\text { communicating }\end{array}$ & $\begin{array}{l}\text { Design implication for } \\
\text { communication } \\
\text { capability }\end{array}$ \\
\hline $\begin{array}{l}\text { Semantic } \\
\text { /Internet }\end{array}$ & $\begin{array}{l}\text { Designed software that give } \\
\text { the liberty to communicate } \\
\text { between things by providing } \\
\text { facility of data translation to } \\
\text { different format (Middleware) }\end{array}$ & $\begin{array}{lr}\text { Things its self } \\
\text { intelligent enough to } \\
\text { communicate and } \\
\text { understand each other }\end{array}$ \\
\hline $\begin{array}{l}\text { Things / } \\
\text { Semantic }\end{array}$ & $\begin{array}{l}\text { Semantically process vast } \\
\text { amount of IoTdata by } \\
\text { scaleable } \\
\text { infrastructure } \\
\text { (Atzori didn't cover this area } \\
\text { well) }\end{array}$ & $\begin{array}{l}\text { Study of data and its } \\
\text { effects on people's } \\
\text { sense making of things }\end{array}$ \\
\hline IoT & $\begin{array}{l}\text { Combination of technologies } \\
\text { that make up IoT }\end{array}$ & $\begin{array}{l}\text { Needed Considerations } \\
\text { at the time of } \\
\text { designing IoT }\end{array}$ \\
\hline
\end{tabular}

Table 1: IOT Framework Comparison

The Things oriented vision includes technical and physical components [20]. In this vision, Varity of everyday objects are introduced, added or upgraded. On the other hand, the Semantic vision addresses the representation, storing, processing, exchange and sharingdata that will involve people in sense making of this data, where semantics is more challenging as a concept and difficult to design. Chetty et al. introduced Home Watch tool to manage home bandwidth. This tool mainly focuses on meeting functional requirements, but lacks the human centered aspects such as managing access to maintain individual privacy and sharing social relations [19].Recent studies and research focus on design HCI some on virtual possessions. The used design may lead to negative outcomes due to the design limitation to handle the variety and massive metadata. The challenge resides in building scalable infrastructure to store vast growing data that can effect on many ways "people sensemaking of things'. So, the Things/Semantic area still immature yet and required more investigation and further research work. 


\section{References}

[1]. Atzori, L., Iera, A., \&Morabito, G. The Internet of Things: a survey. Computer Networks, 54 (2010), 2787-2805.

[2]. Treffyn et al." Approaching a human-centered internet of things". 25th Australian Computer-Human Interaction Conference: Augmentation, Application, Innovation, Collaboration ACM, New York, NY, USA, 363-366.

[3]. C.P. Mayer. Security and Privacy Challenges in the Internet of Things. KiVS Workshop on Global Sensor Network, 2009.

[4]. P. Tavel. Modeling and simulation design. 2007.

[5]. Next Generation Mobile Networks

http://www.ngmn.org/fileadmin/ngmn/content/images/news/ngmn_news/NGMN_5G_White_Paper_V1_0.pdf

[6]. High Availability on Wikipediahttps://en.wikipedia.org/wiki/High_availability

[7]. The METIS 2020 Project - Laying the foundation of 5G https://www.metis2020.com

[8]. EVARILOS EU Projecthttp://www.evarilos.eu/index.php

[9]. CREW EU Projecthttp://www.crew-project.eu/

[10]. 5G: The Internet for Everyone and Everything (NI) http://www.ni.com/pdf/company/en/Trend_Watch_5G.pdf

[11]. How 5G will Power the Future Internet of Things - iQ by Intel http://iq.intel.com/how-5g-will-power-the-future-internet-of-things/

[12]. J. Claessens. Trust, Security, Privacy, and Identity perspective. Panel on Future Internet Service Offer, 2008.

[13]. R. Roman et al. "Integrating Wireless Sensor Networks and the Internet" a Security Analysis. Internet Research, Vol. 19, no. 2, 2009.

[14]. D. Christin et al. "Wireless Sensor Networks and the Internet of Things: Selected Challenges." 8th GI/ITG KuVSFachgesprch 2009.

[15]. A. Kansalet al, "SenseWeb: An Infrastructure for Shared Sensing", IEEE Multimedia, Vol. 14, no. 4, 2007.

[16]. F. Bonomi, "Connected vehicles, the internet of things, and fog computing" Vehicular Ad hoc Networks (VANET) 2011.

[17]. G. Forman. An extensive empirical study of feature selection metrics for text classi_cation. J. Mach. Learn. Res., 3:1289\{1305, Mar. 2003

[18]. M. J. Sannella. Constraint satisfaction and debugging for interactive user interfaces. 2003.

[19]. M. Chetty et al, "Who's hoggingthe bandwidth?: the consequences of revealing the invisible in the home” . ACM press 2010, 659668

[20]. Shahbaz Pervez, Nasser Abosaq, Gasim Alandjani, "IOT Services Impact as a Driving Force on Future Technologies by Addressing Missing Dots", 16th International Conference on Applied Computer Science (ACS '16), Istanbul, Turkey, 15-17 April 2016.

[21]. Shahbaz Pervez, Faheem Babar, Gasim Alandjani, "An Efficient Cloud Model with integrated Services by addressing Major Security Challenges., Journal of World Scientific Engineering Assembly and Society Transactions on Computers Print ISSN: 1109-2750, E-ISSN: 2224-2872

[22]. Shahbaz Pervez, Gasim Alandjani, QurratUlAin, "Internet Traffic Trends and Major Security Challenges in Local \& Internet Cloud" , 19th International Conference on Circuits, Systems, Communications and Computer" in Zakynthos, Greece, July 16-20, 2015.

[23]. Shahbaz Pervez, Nasser Abosaq, RabiaShaheen, Faheem babar, "Mobile Applications Architecture and their Use in cutting-Edge Technologies", International Journal of Information Technology and Electrical Engineering. ISSN: - 2306-708X, April 2, 2014.

[24]. Shahbaz Pervez, Adeel Akram, "A Temporally Efficient Solution for Optimal Wireless Mesh Backbone Device placement and Antenna selection in Wireless Mesh Networks", INFOR MATION (An International Interdisciplinary Journal) ISSN 134 3-4500 ISSN 1344-8994(electronic, January 18, 2012. 